$\begin{array}{ll}\text { Research Square } & \begin{array}{l}\text { Preprints are preliminary reports that have not undergone peer review. } \\ \text { They should not be considered conclusive, used to inform clinical practice, } \\ \text { or referenced by the media as validated information. }\end{array}\end{array}$

\title{
Impact of Type, Dose and Duration of Oral Polyunsaturated Fatty Acid Supplementation on Disease Activity in Inflammatory Rheumatic Diseases: A Systematic Literature Review and Meta-Analysis
}

\author{
Johanna Sigaux ( $\nabla$ johanna.sigaux@aphp.fr) \\ Hopital Avicenne https://orcid.org/0000-0002-6371-740X \\ Sylvain Mathieu \\ CHU Gabriel Montpied: Centre Hospitalier Universitaire Gabriel Montpied \\ Yann Nguyen \\ Hospital Beaujon: Hopital Beaujon \\ Pauline Sanchez \\ Montpellier 1 University: Universite de Montpellier \\ Jean-Guillaume Letarouilly \\ Lille University School of Medicine: Universite de Lille Faculte de Medecine \\ Martin Soubrier \\ CHU Gabriel Montpied: Centre Hospitalier Universitaire Gabriel Montpied \\ Sébastien Czernichow \\ HEGP: Hopital Europeen Georges Pompidou \\ René-Marc Flipo \\ Lille University School of Medicine: Universite de Lille Faculte de Medecine \\ Jérémie Sellam \\ Hospital Saint-Antoine: Hopital Saint-Antoine \\ Claire Daïen \\ University of Montpellier: Universite de Montpellier
}

\section{Research article}

Keywords: Inflammatory rheumatic diseases, Rheumatoid arthritis, Polyunsaturated fatty acids, Omega-3, Omega-6, Meta-analysis, Systematic literature review

Posted Date: November 9th, 2021

DOI: https://doi.org/10.21203/rs.3.rs-1030187/v1

License: @ (i) This work is licensed under a Creative Commons Attribution 4.0 International License. Read Full License 


\section{Abstract}

Background. Polyunsaturated fatty acids (PUFA) supplementation has been reported to improve disease activity in inflammatory rheumatic diseases (IRD). However, data are often conflicting and studies insufficiently large to draw conclusions. This systematic literature review and meta-analysis aimed to better estimate the effect of oral supplementation with omega (n)-3 and n-6 PUFA on IRD activity in terms of duration, dose, type and source.

Methods. The literature was searched in PubMed, EMBASE and Cochrane Library databases up to October 2020. Studies were reviewed in accordance with PRISMA guidelines. The effect of PUFA supplementation on disease activity was expressed as the standardized mean difference (95\% $\mathrm{Cl}$ ). Metaregression and subgroup analyses involved type of IRD, Jadad score, PUFA source (animal or vegetable) and doses.

Results. We obtained 42 references; 30 randomized controlled studies were included comparing the effects of PUFA versus control on disease activity (710 IRD patients receiving PUFA supplementation and 710 controls, most with rheumatoid arthritis). We found a significant improvement in pain, swollen and tender joint count, Disease Activity Score in 28 joints, and Health Assessment Questionnaire score in IRD patients receiving PUFA supplementation as compared with controls, with a significant decrease in erythrocyte sedimentation rate but not C-reactive protein level. Although meta-regression revealed no difference by IRD type or source or dose of PUFA supplementation, subgroup analysis revealed more parameters significantly improved with animal- than vegetable-derived PUFAs and 3-to 6-month supplementation. Most studies examined high-dose supplementation (>2 g/day).

Conclusion. PUFA consumption, especially omega-3 from animal source $>2 \mathrm{~g} /$ day, may improve IRD activity and might be an adjuvant therapy in rheumatoid arthritis.

Trial registration. The protocol was registered at PROSPERO (CRD42021253685).

\section{Background}

In the last 20 years, knowledge about rheumatoid arthritis (RA) and other inflammatory rheumatic diseases (IRDs) has greatly improved and led to effective treatments. Nevertheless, patients' unmet needs remain substantial, and a growing number of patients require alternative therapies as a complement to traditional biological and non-biological disease-modifying anti-rheumatic drugs. Diet is now considered a major component of chronic diseases, especially in RA (1). Nutrition plays a role in the emergence of these diseases as well as their evolution and activity $(2,3)$. Nevertheless, studying the potential effects of diet remains a great challenge because of the complexity and variety of the components, and studies are often disparate in quality and methods. Unlike trials of Mediterranean, vegan and gluten-free diets, randomized controlled trials (RCTs) of supplementation with n-3 polyunsaturated fatty acids (n-3 PUFAs) in patients with IRD, especially encapsulated oil, have been of high quality. Therefore, studies of n-3 PUFA supplementation, mostly derived from fish oil, are the most numerous and are also supported by a pathophysiological rationale, because $n-3$ PUFAs have anti-inflammatory effects (4) and promote immuneregulation activities (5).

Little is known about the effect of n-6 PUFAs. N-6 PUFAs are considered pro-inflammatory components because linoleic acid (LA), included in more than 50\% of the most-consumed vegetable oils, is a precursor of arachidonic acid, itself a precursor of inflammatory mediators (6). Nevertheless, some n-6 PUFAs may have beneficial effects on health. For example, GLA (gamma-linolenic acid), found in certain oils such as evening primrose oil, may have an anti-inflammatory action due to the production of prostaglandin $\mathrm{E}_{1}$ and other eicosanoids (7). Moreover, it has been shown that GLA supplementation decreases ex vivo production of leukotriene $B_{4}$ by neutrophils (8). However, few RCTs have investigated the effect of n-6 PUFA supplementation on IRD activity.

Five heterogenous meta-analyses investigating the effect of n-3 supplementation on disease activity have been published since 1995 (9-13). Three included exclusively RA patients $(9,11,13)$ and one, published in 2012, focused on oral $\mathrm{n}-3$ PUFA supplementation> $2.7 \mathrm{~g} / \mathrm{d}$ (11). The results are broadly consistent with a beneficial effect of n-3 PUFA supplementation on tender joint count (TJC), pain, morning stiffness (MS) and non-steroidal anti-inflammatory drug (NSAID) use. Conversely, the effects on swollen joint count (SJC), inflammatory biomarkers, Health Assessment Questionnaire (HAQ) score and Disease Activity Score in 28 joints (DAS28) are still equivocal.

We performed an updated meta-analysis and meta-regression (by type of IRD, Jadad quality score, PUFA source [animal or vegetable] and dose) of published studies or abstracts. We aimed to update previous meta-analyses with recent literature and better estimate the effect of oral n-3 and n-6 PUFA supplementation by dose and source on disease activity and pain in patients with IRD. This work was conducted to inform the recommendations of the French Society of Rheumatology on dietary practices in IRD.

\section{Methods}

A systematic review of the published literature was performed in accordance with the Preferred Reporting Items for Systematic Reviews and Meta-analyses (PRISMA) guidelines (14).

\section{Data sources and search strategy}

We searched PubMed, Cochrane library and EMBASE databases for English or French reports published up to October 1, 2020. All reports of RCTs exploring the effect of n-3 PUFAs (eicosapentaenoic acid [EPA], docosahexaenoic acid [DHA], docosapentaenoic acid [DPA], alpha Linolenic acid [ALA]) and n-6 PUFAs ( $L A, G L A)$ on disease activity, systemic inflammation or pain over time compared to controls were included. We used the following search terms in PubMed, Cochrane Library and EMBASE databases: ("Fatty Acids, Omega-3" OR "omega 3" OR "n-3 Fatty Acids" OR "n-3 Polyunsaturated Fatty Acid" OR "n-3 PUFA" OR "omega 3 Fatty Acids" OR "Fatty Acids, Omega-6" OR "omega 6" OR "n-6 Fatty Acids" OR "n-6 Polyunsaturated Fatty Acid" OR "n-6 PUFA" OR "Omega 6 Fatty Acids" OR "Polyunsaturated Fatty Acids") AND ("Spondylitis, Ankylosing" OR ankylosis OR Spondylarthritis OR "Arthritis, Rheumatoid" OR "rheumatoid arthritis" 
OR "Arthritis, Psoriatic" OR "Psoriatic Arthritis"). We searched the reference lists of articles. We also collected data from the electronic abstract databases of the annual scientific meetings of the European Alliance of Associations for Rheumatology and American College of Rheumatology from 2009 to 2020 by using the term "omega-3", "omega-6" or "PUFA".

\section{Study selection}

The articles identified were evaluated independently by two investigators (JS and SM) after reading the title, keywords, abstract, then the full text. Any doubts about article selection were resolved by consensus after discussion with other investigators (CD and SM). The studied population comprised patients with rheumatic diseases (RA, ankylosing spondylitis [AS], psoriatic arthritis [PsA]); the intervention analysed was oral supplementation of n-3 and/or $n-6$ PUFAs; the outcomes retained were parameters of disease activity (duration of MS, visual analogue scale [VAS] score for pain and activity; DAS28; TJC, SJC, HAQ score, erythrocyte sedimentation rate [ESR] and C-reactive protein [CRP] level). Only RCTs were included. We excluded citations with an unavailable full-text article and for meta-analysis, those with data not suitable for statistical analysis (no standard deviation or interquartile range for means or medians or intervention not limited to oral PUFA supplementation).

\section{Data extraction}

Two investigators (SM and JS) independently examined participants (patients and control) characteristics and extracted all data by using a standardized data abstraction form. For all extracted data, the number of participants, or a central value (mean or median) and variability (standard deviation or interquartile range) were obtained.

We extracted the number of patients who used PUFAs and the number of controls. For these populations, we extracted the type of rheumatic disease (RA, AS or PSA), the mean change in MS duration, VAS pain and activity, DAS28, TJC, SJC, HAQ score, ESR and CRP level after the intervention.

We also collected data on age and percentage of women. Interventions were classified by source of PUFA (animal or vegetable), type of n-3 PUFAs (EPA, DHA, DPA, ALA) and n-6 PUFAs (LA, GLA), and dose and duration of supplementation. Any marine oil (such as seal oil, microalgae oil) was classified as "fish oil".

\section{Quality assessment of the included studies}

Risk of bias assessments were performed during data collection independently by two authors (SM and JS) and involved the Cochrane Collaboration's Risk of Bias tool and the Jadad scale for each study. Records limited to abstracts were not assessed because of the paucity of available information. We classified the studies according to Jadad score: $\geq 4$ or $<4$.

\section{Statistical analyses}

Baseline characteristics are summarized for each study sample and reported as the mean (standard deviation) or number (percentage) for continuous and categorical variables, respectively. The differences between the effect of n-3/n-6 PUFA supplementation and control on variables of disease activity over time were calculated as the standardized mean difference (SMD) by using the inverse-variance method. We chose the SMD because included studies could assess the same outcome but measure it in different ways. For example, TJC corresponded to the number of painful joints in recent studies and to Richie's index in older studies. The SMD was interpreted according to the Cohen's $d$ as trivial, $<0.2$; small, 0.2-0.3; moderate, 0.5-0.8; and large, >0.8 (15).

Statistical heterogeneity was assessed by examining forest plots, SMD, confidence intervals (Cls), and calculating the $\mathrm{I}^{2}$ index, the most common metric for measuring the magnitude of between-study heterogeneity (16). $I^{2}$ values range from $0-100 \%$ and are considered low at $<25 \%$, modest at $25-50 \%$, and high at $>50 \%$. Random-effects models assuming between-and within-study variability (DerSimonian and Laird approach) were used if heterogeneity was present; otherwise, a fixed-effects model was used. When possible (sufficient sample size), meta-regression analysis was used to study the relation between effect sizes of n-3/n-6 PUFA consumption, SMDs of TJC and SJC, VAS pain, HAQ score, DAS28, CRP level and ESR, MS duration and study characteristics (Jadad score $\geq 4$ or $<4$, type, source and dose of PUFA and type of rheumatic disease). Subgroup analyses were also performed according to these characteristics.

Finally, to check the robustness of the results, we performed sensitivity analyses excluding studies not evenly distributed around the base of the funnel plots and according to Egger's test (publication bias). Two-sided $p<0.05$ was considered statistically significant. All statistical analyses involved using Stata 15 (StataCorp, College Station, TX, USA). The PRISMA checklist is in the appendix (Additional file 1). The protocol was registered at PROSPERO (CRD42021253685).

\section{Results}

A total of 1630 citations were obtained from the initial search and screened for titles and abstracts (Fig. 1).

After excluding articles not meeting the inclusion criteria, 117 full papers were screened and 42 were included in the systematic review. Among them, 30 had sufficient data and were included in the meta-analysis. Characteristics of the included studies are presented in Table 1 (and Additional file 2). 
Table 1

Characteristics of the publications included in the meta-analysis by source and type of polyunsaturated fatty acid (PUFA)

\begin{tabular}{|c|c|c|c|c|c|c|c|c|c|c|c|c|c|c|}
\hline \multirow[t]{2}{*}{ Study } & \multirow[t]{2}{*}{ Disease } & \multicolumn{2}{|c|}{$\begin{array}{l}\text { No. of } \\
\text { participants }\end{array}$} & \multirow[t]{2}{*}{$\begin{array}{l}\text { PUFA } \\
\text { source }\end{array}$} & \multicolumn{7}{|c|}{ PUFAs dose (g/day) } & \multirow[t]{2}{*}{ Control } & \multirow[t]{2}{*}{$\begin{array}{l}\text { Duration } \\
\text { (weeks) }\end{array}$} & \multirow[t]{2}{*}{$\begin{array}{l}\mathrm{Ji} \\
\text { si }\end{array}$} \\
\hline & & PUFA & Total & & EPA & DHA & DPA & ALA & $\begin{array}{l}\text { Total } \\
\text { n-3 } \\
\text { intake }\end{array}$ & GLA & $\begin{array}{l}\text { Total } \\
\mathrm{n}-6 \\
\text { intake }\end{array}$ & & & \\
\hline $\begin{array}{l}\text { Araujo } \\
2014(17)\end{array}$ & RA & 11 & 37 & Fish oil & & & & & & & & Not mentioned & 24 & at \\
\hline $\begin{array}{l}\text { Berbert } \\
2005 \text { (18) }\end{array}$ & RA & 30 & 43 & Fish oil & & & & & 3 & & & Soy oil & 24 & 2 \\
\hline $\begin{array}{l}\text { Brzeski } \\
1991(19)\end{array}$ & RA & 19 & 40 & $\begin{array}{l}\text { Vegetable } \\
\text { oil }\end{array}$ & & & & & & 0.5 & 0.5 & Olive oil capsules & 24 & 3 \\
\hline $\begin{array}{l}\text { Cleland } \\
1988(20)\end{array}$ & RA & 23 & 23 & Fish oil & & & & & 5.2 & & & Olive oil capsules & 12 & 4 \\
\hline $\begin{array}{l}\text { Das Gupta } \\
2009(21)\end{array}$ & RA & 50 & 100 & Fish oil & & & & & 3 & & & $\begin{array}{l}\text { No special diet or } \\
\text { intervention }\end{array}$ & 12 & 1 \\
\hline $\begin{array}{l}\text { Dawczynski } \\
2009(22)\end{array}$ & RA & 14 & 26 & Fish oil & 0.7 & 0.4 & 0.1 & 1.1 & 2.3 & & & $\begin{array}{l}\text { Dairy with comparable } \\
\text { fat content }\end{array}$ & 24 & 3 \\
\hline $\begin{array}{l}\text { Dawczynski } \\
2011(23)\end{array}$ & PsA+RA & 14 & 47 & Fish oil & & & & & 3.0 & & & Olive oil capsules & 12 & 4 \\
\hline $\begin{array}{l}\text { Dawczynski } \\
2018(24)\end{array}$ & RA & 25 & 25 & Fish oil & & 2.1 & & & 2.1 & & & Sunflower oil capsules & 10 & 3 \\
\hline $\begin{array}{l}\text { Galarraga } \\
2008(25)\end{array}$ & RA & 49 & 97 & Fish oil & 1.5 & 0.7 & & & 2.2 & & & Air-filled capsules & 36 & 4 \\
\hline \multirow{2}{*}{$\begin{array}{l}\text { Geusens } \\
1994(26)\end{array}$} & \multirow[t]{2}{*}{ RA } & 21 & \multirow[t]{2}{*}{60} & \multirow[t]{2}{*}{ Fish oil } & & & & & 1.3 & & & \multirow[t]{2}{*}{ Olive oil capsules } & \multirow[t]{2}{*}{48} & \multirow[t]{2}{*}{3} \\
\hline & & 19 & & & & & & & 2.6 & & & & & \\
\hline $\begin{array}{l}\text { Khaleghi } \\
2005(27)\end{array}$ & RA & 10 & 39 & Fish oil & & & & & 10 & & & Corn oil & 8 & al \\
\hline $\begin{array}{l}\text { Kolahi } 2010 \\
(28)\end{array}$ & RA & 40 & 83 & Fish oil & 0.2 & 0.1 & & & 0.3 & & & $\begin{array}{l}\text { Capsules without n-3 } \\
\text { PUFAs }\end{array}$ & 24 & 3 \\
\hline \multirow{2}{*}{$\begin{array}{l}\text { Kremer } \\
1990(29)\end{array}$} & \multirow[t]{2}{*}{ RA } & 20 & \multirow[t]{2}{*}{49} & \multirow[t]{2}{*}{ Fish oil } & 1.9 & 1.3 & & & 3.2 & & & \multirow[t]{2}{*}{ Olive oil capsules } & \multirow[t]{2}{*}{24} & \multirow[t]{2}{*}{2} \\
\hline & & 17 & & & 3.8 & 2.5 & & & 6.3 & & & & & \\
\hline $\begin{array}{l}\text { Kremer } \\
1995(30)\end{array}$ & RA & 23 & 49 & Fish oil & & & & & 9.1 & & & Corn oil capsules & 30 & 2 \\
\hline $\begin{array}{l}\text { Kremer } \\
1985(31)\end{array}$ & RA & 23 & 44 & Fish oil & 1.7 & 1.1 & & & 2.8 & & & $\begin{array}{l}\text { Diet low in PUFA/SFA } \\
\text { plus paraffin capsules }\end{array}$ & 12 & 4 \\
\hline $\begin{array}{l}\text { Kristensen } \\
2018(32)\end{array}$ & PsA & 72 & 143 & Fish oil & 1.5 & 1.5 & & & 3.0 & & & Olive oil capsules & 24 & 5 \\
\hline $\begin{array}{l}\text { Leventhal } \\
1993(33)\end{array}$ & RA & 19 & 37 & $\begin{array}{l}\text { Vegetable } \\
\text { oil }\end{array}$ & & & & & & 1.4 & 1.4 & Cottonseed oil capsules & 24 & 2 \\
\hline $\begin{array}{l}\text { Madland } \\
2006(34)\end{array}$ & PsA & 20 & 40 & Fish oil & 2.4 & 2.6 & 1.1 & & 6.1 & & & Soil oil capsules & 0.5 & 2 \\
\hline $\begin{array}{l}\text { Magaro } \\
1992(35)\end{array}$ & RA & 10 & 20 & Fish oil & 1.5 & 1.0 & & & 2.5 & & & $\begin{array}{l}\text { No special diet or } \\
\text { intervention }\end{array}$ & 6 & 1 \\
\hline $\begin{array}{l}\text { Nielsen } \\
1992(36)\end{array}$ & RA & 29 & 57 & Fish oil & 2.0 & 1.2 & & & 3.2 & & & $\begin{array}{l}\text { Capsules with fat } \\
\text { composition as the } \\
\text { average Danish diet }\end{array}$ & 12 & 4 \\
\hline
\end{tabular}




\begin{tabular}{|c|c|c|c|c|c|c|c|c|c|c|c|c|c|c|}
\hline \multirow{2}{*}{$\begin{array}{l}\text { Study } \\
\text { Nordström } \\
1995 \text { (37) }\end{array}$} & \multirow{2}{*}{$\begin{array}{l}\text { Disease } \\
\text { RA }\end{array}$} & \multicolumn{2}{|c|}{$\begin{array}{l}\text { No. of } \\
\text { participants }\end{array}$} & \multirow{2}{*}{$\begin{array}{l}\begin{array}{l}\text { PUFA } \\
\text { source }\end{array} \\
\begin{array}{l}\text { Vegetable } \\
\text { oil }\end{array}\end{array}$} & \multicolumn{5}{|c|}{ PUFAs dose (g/day) } & & & \multirow{2}{*}{$\begin{array}{l}\text { Control } \\
\text { Safflower oil }\end{array}$} & \multirow{2}{*}{$\begin{array}{l}\begin{array}{l}\text { Duration } \\
\text { (weeks) }\end{array} \\
24\end{array}$} & \multirow[t]{2}{*}{$\begin{array}{ll}\text { Ji } \\
\text { si }\end{array}$} \\
\hline & & 11 & 22 & & & & & 9.6 & 9.6 & & & & & \\
\hline $\begin{array}{l}\text { Park } 2013 \\
\text { (38) }\end{array}$ & RA & 41 & 81 & Fish oil & 2.1 & 1.2 & & & 3.3 & & & $\begin{array}{l}\text { sunflower oil with oleic } \\
\text { acid capsules }\end{array}$ & 16 & 2 \\
\hline $\begin{array}{l}\text { Skoldstam } \\
1992(39)\end{array}$ & RA & 22 & 46 & Fish oil & 1.7 & 1.1 & & & 2.8 & & & $\begin{array}{l}\text { Maize/olive/peppermint } \\
\text { oil capsules }\end{array}$ & 24 & 4 \\
\hline $\begin{array}{l}\text { Sundrarjun } \\
2004(40)\end{array}$ & RA & 23 & 60 & Fish oil & & & & & 3.4 & & & $\begin{array}{l}\text { Diet low in PUFA plus } \\
\text { placebo capsules }\end{array}$ & 24 & 3 \\
\hline $\begin{array}{l}\text { Sundström } \\
2006(41)\end{array}$ & AS & 9 & 18 & Fish oil & & & & & 1.9 & & & Low dose vs high dose & 21 & 1 \\
\hline $\begin{array}{l}\text { Tulleken } \\
1990 \text { (42) }\end{array}$ & RA & 12 & 24 & Fish oil & 2.0 & & 1.3 & & 3.3 & & & Coconut oil capsules & 24 & 4 \\
\hline $\begin{array}{l}\text { Veale } 1994 \\
(43)\end{array}$ & PsA & 19 & 38 & Mix oil & 0.2 & 0.1 & & & 0.3 & 0.5 & 0.5 & $\begin{array}{l}\text { Liquid paraffin and } \\
\text { vitamin E capsules }\end{array}$ & 36 & 2 \\
\hline $\begin{array}{l}\text { Veselinovic } \\
2017 \text { (44) }\end{array}$ & RA & 20 & 60 & Fish oil & 1.0 & 1.5 & & & 2.5 & & & $\begin{array}{l}\text { No special diet or } \\
\text { intervention }\end{array}$ & 12 & 2 \\
\hline $\begin{array}{l}\text { Volker } 2000 \\
\text { (45) }\end{array}$ & RA & 13 & 26 & Fish oil & & & & & 1.7 & & & $\begin{array}{l}\text { Diet low in u-6 PUFAs } \\
\text { plus corn and olive oil }\end{array}$ & 15 & 3 \\
\hline $\begin{array}{l}\text { Zurier } 1996 \\
(46)\end{array}$ & RA & 28 & 56 & $\begin{array}{l}\text { Vegetable } \\
\text { oil }\end{array}$ & & & & & & 2.8 & 2.8 & Sunflower seed oil & 24 & 4 \\
\hline
\end{tabular}

EPA= eicosapentaenoic acid (C20:5n3); DHA= docosahexaenoic acid (C22:6n3); DPA= docosapentaenoic acid (C22:5n3); ALA= Linolenic acid (C18:3n3); GLA= Gamma-linolenic acid (C18:3n6); n-3= omega-3; n-6= omega-6; PUFA= polyunsaturated fatty acid; $E P O=$ evening primrose oil; RA= rheumatoid arthritis; PsA= psoriatic arthritis; $A S=$ ankylosing spondylitis; activity= patient activity on visual analogue scale; $\mathrm{CRP}=\mathrm{C}$-reactive protein; $\mathrm{DAS}$ : disease activity score; $\mathrm{ESR}=$ erythrocyte sedimentation rate; $\mathrm{HAQ}=$ Health Assessment Questionnaire; MS= morning stiffness; NSAID= non-steroidal anti-inflammatory drugs; $\mathrm{SJ}=$ swollen joint count; $\mathrm{TJ}=$ tender joint count

The assessment of quality of the 40 included studies (2 abstracts (17) and (27)) were excluded from the quality analysis) revealed a selective reporting bias. The primary outcome was rarely defined in these studies (risk of bias according to the Cochrane Collaboration Risk of Bias tool summarized in Additional file 3). The median Jadad score for these 40 studies was 3 (interquartile range 2-4). Twelve (30 \%) studies had a Jadad score $\geq 4$.

We included 30 of the 42 studies in the meta-analysis (Table 1) (710 patients receiving PUFAs and 710 controls); 10 studies were excluded because data were lacking on standard deviation, mean or number of patients included (Additional file 2). Despite an e-mail sent to authors, we did not obtain usable data for these studies. Two other studies were excluded because of mixed supplementation of n-3/n-6 PUFAs and zinc or other nutrients (Additional file 2).

At baseline, the mean age of the 710 patients starting PUFA supplementation was 53.3 years, $73.5 \%$ were women and disease activity was high (weighted mean \pm sd TJC: $12.2 \pm 6.3$, SJC: $7.8 \pm 3.3$, DAS28: $4.3 \pm 0.7$, CRP level: $16.4 \pm 11.1 \mathrm{mg} / \mathrm{l})$. The characteristics of patients included in the meta-analysis are presented in Additional file 4. Disease duration and baseline disease activity were globally similar between intervention and control groups.

\section{Overall effects of PUFA supplementation on disease activity}

When pooling all studies and timepoints, all clinical parameters of disease activity were significantly improved by oral PUFA supplementation. Improvement was high for VAS activity; moderate for VAS pain, DAS28 and HAQ score; and low for MS duration, SJC and TJC (Table 2). 
Table 2

Effect of oral PUFA supplementation on parameters of inflammatory rheumatic disease activity compared to control

\begin{tabular}{|c|c|c|c|c|c|}
\hline \multirow[t]{2}{*}{ Parameters } & \multicolumn{4}{|c|}{ Effect of PUFA supplementation over time } & \multirow[t]{2}{*}{$1^{2}$} \\
\hline & After 1 month of treatment & After 3 months of treatment & After 6 months of treatment & Overall effect* & \\
\hline \multirow[t]{3}{*}{ Tender joints } & $N=3$ & $\mathrm{~N}=15$ & $\mathrm{~N}=9$ & $-0.31[-0.52,-0.10]$ & $67 \% R$ \\
\hline & $n=122(62+60)$ & $\mathrm{n}=606(305+301)$ & $\mathrm{n}=408(206+202)$ & $p=0.004$ & \\
\hline & $-0.34[-0.75,0.08]$ & $-0.26[-0.57,0.05]$ & $-0.39[-0.78,-0.000]$ & & \\
\hline \multirow[t]{3}{*}{ Swollen joints } & $N=3$ & $N=11$ & $\mathrm{~N}=7$ & $-0.22[-0.39,-0.05]$ & $39 \%$ \\
\hline & $n=122(62+60)$ & $\mathrm{n}=467(237+230)$ & $\mathrm{n}=330(168+162)$ & $p=0.01$ & $\mathrm{R}$ \\
\hline & $-0.09[-0.70,0.51]$ & $-0.32[-0.55,-0.09]$ & $-0.11[-0.42,0.19]$ & & \\
\hline \multirow[t]{3}{*}{ Morning stiffness duration } & $\mathrm{N}=2$ & $\mathrm{~N}=11$ & $N=6$ & $-0.38[-0.61,-0.15]$ & $56 \% R$ \\
\hline & $\mathrm{n}=56(29+27)$ & $n=509(258+251)$ & $\mathrm{n}=192(100+92)$ & $p=0.001$ & \\
\hline & $-0.47[-1.60,0.66]$ & $-0.19[-0.36,-0.01]$ & $-0.77[-1.39,-0.16]$ & & \\
\hline \multirow[t]{3}{*}{ VAS pain } & $N=3$ & $\mathrm{~N}=13$ & $\mathrm{~N}=11$ & $-0.48[-0.95,-0.01]$ & $93 \% \mathrm{R}$ \\
\hline & $n=106(53+53)$ & $n=597(300+297)$ & $n=549(279+270)$ & $p=0.04$ & \\
\hline & $-1.46[-4.33,1.41]$ & $-0.57[-0.99,-0.15]$ & $-0.32[-1.24,0.60]$ & & \\
\hline \multirow[t]{3}{*}{ VAS activity } & $\mathrm{N}=2$ & $N=7$ & $N=8$ & $-1.05[-1.63,-0.47]$ & $91 \% \mathrm{R}$ \\
\hline & $n=86(43+43)$ & $n=266(136+130)$ & $\mathrm{n}=280(143+137)$ & $P<0.001$ & \\
\hline & $-1.04[-2.47,0.38]$ & $-1.08[-2.16,-0.009]$ & $-1.04[-1.92,-0.16]$ & & \\
\hline \multirow[t]{3}{*}{ DAS28 } & & $N=7$ & $N=3$ & $-0.36[-0.60,-0.11]$ & $58 \% \mathrm{R}$ \\
\hline & & $n=419(209+210)$ & $\mathrm{n}=255(126+129)$ & $p=0.005$ & \\
\hline & & $-0.41[-0.78,-0.04]$ & $-0.22[-0.47,0.02]$ & & \\
\hline \multirow[t]{3}{*}{ CRP } & $\mathrm{N}=1$ & $\mathrm{~N}=13$ & $N=5$ & $-0.08[-0.53,0.36]$ & $90 \% \mathrm{R}$ \\
\hline & $\mathrm{n}=46(23+23)$ & $n=669(335+334)$ & $\mathrm{n}=238(118+120)$ & $p=0.71$ & \\
\hline & $-0.52[-1.10,0.07]$ & $0.15[-0.44,0.74]$ & $-0.62[-1.17,-0.06]$ & & \\
\hline \multirow[t]{3}{*}{ ESR } & $\mathrm{N}=3$ & $\mathrm{~N}=11$ & $N=6$ & $-0.28[-0.43,-0.13]$ & $44 \% \mathrm{R}$ \\
\hline & $n=106(53+53)$ & $\mathrm{n}=426(214+212)$ & $\mathrm{n}=186(92+94)$ & $P<0.001$ & \\
\hline & $-0.47[-0.86,-0.08]$ & $-0.25[-0.50,0.002]$ & $-0.23[-0.52,0.06]$ & & \\
\hline \multirow[t]{3}{*}{ HAQ } & $\mathrm{N}=2$ & $\mathrm{~N}=3$ & $\mathrm{~N}=4$ & $-0.66[-1.11,-0.21]$ & $85 \% R$ \\
\hline & $n=86(43+43)$ & $\mathrm{n}=204(103+101)$ & $\mathrm{n}=316(160+156)$ & $p=0.004$ & \\
\hline & $-0.15[-0.93,0.63]$ & $-1.48[-2.92,-0.04]$ & $-0.48[-0.87,-0.09]$ & & \\
\hline
\end{tabular}

Data are standarized mean difference [95\% confidence interval].

VAS= visual analogue scale; DAS28= Disease Activity Score in 28 joints; $\mathrm{ESR}=$ erythrocyte sedimentation rate; $\mathrm{CRP}=\mathrm{C}$-reactive protein; $\mathrm{HAQ}=\mathrm{Health}$ Assessment Questionnaire

$\mathrm{N}=$ number of studies; $\mathrm{n}=$ number of patients

$n=Z(X+Y): Z=$ number of participants $(X=$ number of patients receiving $P U F A s+Y=n u m b e r$ of controls)

$\mathrm{R}=$ random effects analysis; $\mathrm{F}=$ fixed effects analysis

*when pooling all studies and timepoints

We found a conflicting effect of oral PUFA supplementation on factors of systemic inflammation, with a significant decrease in ESR but not CRP level (Table 2). Similar results were found when including only RA patients, who represented most of the studied population (26/30 studies: $1192 / 1420$ patients) (Additional file 5, Figure 2, Additional file 6 to 14).

Sensitivity analyses according to funnel plots and metabias revealed no difference in MS duration or VAS activity after excluding Kremer et al. (31) and Geusens et al. (26) (Additional file 15). Likewise, Egger's test was statistically significant for CRP analysis ( $p=0.047$ ) because of the results of the Das Gupta et 
al. study (21). Sensitivity analysis excluding this study revealed a significant improvement in CRP level (overall pooled SMD for CRP = -0.37 [95\%Cl $-0.60 ;-0.13$; $\mathrm{p}=0.002$ ). Furthermore, meta-regression revealed no significant effect of overall results for the Jadad score (Fig. 3).

Of the studies included in the systematic review but not in the meta-analysis, only 4 out of 10 and 2 out of 4 showed a significant positive effect (or a trend) for TJC and SJC, respectively. All positive studies included high doses of n-3 PUFAs ( $2 \mathrm{~g}$ per day) and/or mussels, as did half of the negative studies. Surprisingly, only one out of 7 studies (mussel-based diet) showed an effect on pain and 3 out of 8 a positive effect on MS; none found significant results regarding ESR (among 6 studies, CRP level (5 studies) and HAQ score (3 studies). Two out of 4 studies showed a significant decrease in DAS28; both included mussels as an n-3 PUFA source (data not shown).

\section{Effect of oral PUFA supplementation on disease activity over time}

None of the clinical and biological parameters were significantly changed after 1 month of supplementation ( 1 to 3 studies, including 46 to 122 patients), whereas all clinical parameters but TJC were significantly improved after 3 months (3 to 15 studies, including 204 to 669 patients) (Table 2). Similarly, when focusing on RA patients, 1-month supplementation significantly decreased only VAS activity (1 to 2 studies, including 46 to 82 patients), whereas 3 -month supplementation significantly decreased all clinical parameters and no biological parameters (ESR, CRP level) (3 to 14 studies, including 204 to 669 patients) (Additional file 5, Fig. 2).

\section{Effect of oral PUFA supplementation on RA disease activity by PUFA type (n-3 or $n-6)$}

Because RA patients represented much of the studied population, to increase homogeneity, we focused a subgroup analysis on studies including RA patients. All but 3 studies used n-3 PUFA supplementation. Analyses focusing on n-3 PUFA supplementation showed a significant improvement in all parameters, except SJC, VAS pain and CRP level (Fig. 4). Supplementation with n-6 PUFAs significantly decreased SJC, VAS activity and HAQ score (Fig. 4).

\section{Effect of oral PUFA supplementation on RA disease activity by}

\section{PUFA source (vegetable or animal)}

Subgroup analysis revealed that animal-derived PUFA supplementation significantly decreased TJC, VAS activity, MS duration, HAQ score, CRP level, and ESR but not SJC, DAS28 or VAS pain (Fig. 5). Conversely, vegetable-derived PUFA supplementation significantly decreased only SJC, VAS activity and HAQ score (Fig. 5).

However, a meta-regression comparing the 2 sources did not show any significant difference (Fig. 3).

\section{Effect of oral PUFA supplementation on RA disease activity by dosage}

Subgroup analysis revealed that high-dose PUFA supplementation ( $\geq 2 \mathrm{~g} /$ day) significantly decreased TJC, VAS activity, MS duration, DA28, HAQ score, and ESR but not SJC, VAS pain or and CRP level (Fig. 6). Low-dose PUFA supplementation (<2 g/day) significantly decreased only CRP level (Fig. 6).

Meta-regression comparing the 2 doses did not show any significant difference (Fig. 3)

\section{Discussion}

The management of IRD is mostly based on a drug-based approach mainly targeting a reduction in inflammation to prevent joint damage, morbidity and mortality. However, patients with these diseases consider the reduction in pain, fatigue and maintenance of physical function as major goals (47-49). Thus, despite increasingly effective management, patients express a significant number of unmet needs, with the need for self-management of the disease, particularly through diet. This meta-analysis included 30 reports of RCTs assessing the effect of oral PUFA supplementation (n-3 and/or n-6, fish and/or vegetable oil) on IRD activity. Oral PUFA supplementation had a beneficial effect on most criteria for disease activity across all IRDs (RA, PsA and AS) even though most studies involved RA patients. Improvement was large for VAS activity; moderate for VAS pain, DAS28 and HAQ level; and small for MS duration, SJC and TJC. These beneficial effects seemed to be observed only for a minimum of 3 months of supplementation because none of the clinical and biological parameters were significantly changed after 1 month of supplementation, whereas all clinical parameters (but TJC) were significantly improved after 3 months and 6 months. As no clinical trials explored supplementation duration above 6 months, we recommend not to extend it any longer. We found a significant decrease in only ESR but not CRP level. Hence, DAS28 improvement seemed linked more to a significant decrease in SJC and VAS activity than a significant improvement in biological systemic inflammation.

\section{Subgroup analyses}

The novelty of this meta-analysis is the subgroup analyses by duration, type, origin and dose of PUFAs. Indeed, for the Lee et al. meta-analysis published in 2012 (11), including studies of only $\geq 2.3-\mathrm{g} / \mathrm{d}$ supplementation (10 RCTs), results were contradictory with previous and subsequent meta-analyses. The Lee et al. meta-analysis found a beneficial effect of supplementation on only NSAID use and no effect on TJC, SJC and MS duration, unlike the meta-analyses of Gioxari et al., Goldberg et al. and Fortin et al. $(9,10,13)$. These results favoured a possible dose-effect of PUFAs with a homeopathic threshold at $2.3 \mathrm{~g} / \mathrm{d}$. Our meta-analysis, which included many more RCTs, found effects on these different parameters that seemed to be independent of PUFA dose. Nevertheless, most studies ( 24 of 29 with available PUFA dose) assessed a dose $>2 \mathrm{~g} / \mathrm{d}$. Thus, the strongest evidence of PUFA efficacy remains with supplementation $>2 \mathrm{~g} / \mathrm{d}$. It is noteworthy that higher dose supplementation has been associated with an increased risk of bleeding and therefore it is recommended not to exceed this dosage $(50,51)$. On subgroup analysis by origin of PUFA (animal or vegetable source), more parameters were significantly improved with fish oil than a 
vegetable source (6/9 vs 3/9). However, most studies concerned fish-oil supplementation $(23 / 31)$ and the difference might also be explained by a lack of power for the vegetable subgroup analysis.

The most recent meta-analysis was published in 2018 (13). It included 20 reports of RCTs that focused exclusively on n-3 PUFA supplementation in RA. The main results showed a significant improvement in TJC, MS duration, pain, HAQ score and ESR, with no effect on CRP level, SJC and DAS28. Therefore, our results are broadly consistent (except for DAS28 and SJC) and strengthen these results with the analysis of 11 additional RCTs.

\title{
Clinical implications
}

These positive results, combined with minor side effects, support the need for nutritional support in addition to standard drug therapy in IRD patients. Beyond the impact on disease activity, the beneficial effects could be cardiovascular as well as for high cardiovascular mortality and morbidity. A recent meta-analysis reported beneficial effects of PUFA supplementation on cardiovascular disease with at least a dose of $2 \mathrm{~g} / \mathrm{d}$ (52). However, as compared with the large evidence available for low doses, the evidence for higher doses (2-4 g/day) is weak and the beneficial effects of $n-3$ PUFAs on cardiovascular risk remain debated (53). The STRENGTH trial of statin-treated participants with high cardiovascular risk, hypertriglyceridemia and low level of high-density lipoprotein cholesterol found no beneficial effect of $4 \mathrm{~g} / \mathrm{d}$ of a carboxylic acid formulation of n-3 PUFAs (a combination of EPA and DHA) as compared with corn oil (53). However, in the same high-risk, statin-treated population, the REDUCE-IT trial found a $25 \%$ reduction in a composite endpoint of cardiovascular events in the n3-treated group (icosapent ethyl) versus the mineral oil-treated group (51). Hence, the effect of PUFA supplementation on cardiovascular events in IRD patients remains to be explored.

\section{Limitations}

Our study has limitations. First, the number of patients with inflammatory rheumatic disease in our meta-analysis was quite low. Most studies included fewer than 30 patients receiving PUFAs, which could be considered not sufficient to draw strong conclusions for the effect on disease activity. However, the inclusion of studies represented more than 700 patients and 700 controls, which is appropriate for a meta-analysis. Moreover, we included only RCTs and performed a subgroup analysis by Jadad score for quality, which revealed no difference in results. Another limitation is related to the publication bias, positive studies being more likely to be published than negative ones. However, we searched for relevant abstracts in European and American congresses and found no other references. Finally, because most studies in the meta-analysis focused on patients with RA, we cannot extrapolate these results to AS and PsA patients. Studies of these specific diseases are needed to increase the level of evidence. These limitations result in a great degree of heterogeneity between studies for some outcomes.

\section{Conclusions}

The findings of this systematic review and meta-analysis suggest that PUFA supplementation, especially omega-3 from animal source $>2 \mathrm{~g} /$ day, seems of interest in terms of disease activity for IRD, with good tolerance and potential additional cardiovascular benefits, in addition to DMARD prescription.

\section{Abbreviations}

\author{
ALA \\ alpha linolenic acid \\ AS \\ ankylosing spondylitis \\ CRP \\ C-reactive protein \\ DAS28 \\ disease activity score in 28 joints \\ DHA \\ docosahexaenoic acid \\ DPA \\ docosapentaenoic acid \\ EPA \\ eicosapentaenoic acid \\ ESR \\ erythrocyte sedimentation rate \\ GLA \\ gamma-linolenic acid \\ HAQ \\ health assessment questionnaire \\ IRD \\ inflammatory rheumatic diseases \\ LA \\ linoleic acid \\ MS
}


morning stiffness

$\mathrm{n}-3 / \mathrm{n}-6$

omega $3 / 6$

NSAID

non-steroidal anti-inflammatory drug

PUFA

polyunsaturated fatty acids

PsA

psoriatic arthritis

RA

rheumatoid arthritis

RCT

randomized controlled trial

SJC

swollen joint count

TJS

tender joint count

VAS

visual analogue scale

\section{Declarations}

Ethics approval and consent to participate: Not applicable.

Consent for publication: Not applicable.

Availability of data and materials: The datasets used and analysed during the current study are available from the corresponding author on reasonable request.

Competing interest: The authors declare no conflicts of interest related to this work.

JSi: Consulting fees: Sandoz, Novartis; SM: none; YN: none; PS: none; JGL: Consulting fees: Sêmeia; MS : none; SC: Consulting fees: Lilly, Janssen, BMS, Fresenius Kabi, Novonordisk, Servier, Bariatek, Fitforme, Mygoodlife; RMF: none; Jse: Consulting fees: Roche, Chugai, Pfizer, BMS, MSD, Biogen, Abbvie, Sandoz, Janssen, Novartis, Fresenius Kabi, Sanofi, Research grants: Pfizer, MSD, Schwa Medico; CD: Consulting fees : Abbvie, Abivax, BMS, Fresenius Kabi, Novartis, Pfizer, Sandoz, Sanofi, Roche- Chugai, UCB.

Funding: This research did not receive any specific grant from funding agencies in the public, commercial, or not-for-profit sectors.

Authors' contribution: JSi: Conceptualization, Data curation, Methodology, Writing- Original draft preparation, Writing - Review \& Editing. SM: Conceptualization, Data curation, Methodology, Writing- Original draft preparation, Writing - Review \& Editing. YNG: Data curation, Writing - Review \& Editing. Pauline Sanchez: Data curation, Writing - Review \& Editing. JGL: Data curation, Writing - Review \& Editing. MS: Visualization, Writing - Review \& Editing, Supervision. SC: Visualization, Writing - Review \& Editing, Supervision. RMF: Visualization, Writing - Review \& Editing. JSe: Conceptualization, Methodology, Writing- Original draft preparation, Writing - Review \& Editing, Supervision. CD: Conceptualization, Methodology, Writing- Original draft preparation, Writing Review \& Editing, Supervision.

Acknowledgements: None.

\section{References}

1. Cassotta M, Forbes-Hernandez TY, Cianciosi D, Elexpuru Zabaleta M, Sumalla Cano S, Dominguez I, et al. Nutrition and Rheumatoid Arthritis in the «Omics » Era. Nutrients. 26 févr 2021;13(3).

2. Nguyen Y, Salliot C, Gelot A, Gambaretti J, Mariette X, Boutron-Ruault M-C, et al. Mediterranean Diet and Risk of Rheumatoid Arthritis: Findings From the French E3N-EPIC Cohort Study. Arthritis Rheumatol. janv 2021;73(1):69-77.

3. Sparks JA, Costenbader KH. Rheumatoid arthritis in 2017: Protective dietary and hormonal factors brought to light. Nat Rev Rheumatol. févr 2018;14(2):71-2.

4. Bäck M, Hansson GK. Omega-3 fatty acids, cardiovascular risk, and the resolution of inflammation. FASEB J. févr 2019;33(2):1536-9.

5. Gutiérrez S, Svahn SL, Johansson ME. Effects of Omega-3 Fatty Acids on Immune Cells. Int J Mol Sci. 11 oct 2019;20(20).

6. Schmitz G, Ecker J. The opposing effects of n-3 and n-6 fatty acids. Prog Lipid Res. mars 2008;47(2):147-55.

7. Johnson MM, Swan DD, Surette ME, Stegner J, Chilton T, Fonteh AN, et al. Dietary supplementation with gamma-linolenic acid alters fatty acid content and eicosanoid production in healthy humans. J Nutr. août 1997;127(8):1435-44.

8. Ziboh VA, Naguwa S, Vang K, Wineinger J, Morrissey BM, Watnik M, et al. Suppression of leukotriene B4 generation by ex-vivo neutrophils isolated from asthma patients on dietary supplementation with gammalinolenic acid-containing borage oil: possible implication in asthma. Clin Dev Immunol. mars 
2004;11(1):13-21.

9. Fortin PR, Lew RA, Liang MH, Wright EA, Beckett LA, Chalmers TC, et al. Validation of a meta-analysis: The effects of fish oil in rheumatoid arthritis. Journal of Clinical Epidemiology. 1 nov 1995;48(11):1379-90.

10. Goldberg RJ, Katz J. A meta-analysis of the analgesic effects of omega-3 polyunsaturated fatty acid supplementation for inflammatory joint pain. Pain. mai 2007;129(1-2):210-23.

11. Lee Y-H, Bae S-C, Song G-G. Omega-3 polyunsaturated fatty acids and the treatment of rheumatoid arthritis: a meta-analysis. Arch Med Res. juill 2012;43(5):356-62.

12. Senftleber NK, Nielsen SM, Andersen JR, Bliddal H, Tarp S, Lauritzen L, et al. Marine Oil Supplements for Arthritis Pain: A Systematic Review and MetaAnalysis of Randomized Trials. Nutrients. 6 janv 2017;9(1).

13. Gioxari A, Kaliora AC, Marantidou F, Panagiotakos DP. Intake of $\omega-3$ polyunsaturated fatty acids in patients with rheumatoid arthritis: A systematic review and meta-analysis. Nutrition. janv 2018;45:114-124.e4.

14. Moher D, Liberati A, Tetzlaff J, Altman DG, PRISMA Group. Preferred reporting items for systematic reviews and meta-analyses: the PRISMA statement. PLoS Med. 21 juill 2009;6(7):e1000097.

15. Cohen J. A power primer. Psychol Bull. juill 1992;112(1):155-9.

16. Higgins JPT, Thompson SG, Deeks JJ, Altman DG. Measuring inconsistency in meta-analyses. BMJ. 6 sept 2003;327(7414):557-60.

17. Araujo AC, Moraes-Fontes M, Santos L, Riso N. Fatty acids and Mediterranean diet as complementary therapies for rheumatoid arthritis. Arthritis Rheum 2014; 66 (Suppl 10):1050.

18. Berbert AA, Kondo CRM, Almendra CL, Matsuo T, Dichi I. Supplementation of fish oil and olive oil in patients with rheumatoid arthritis. Nutrition. févr 2005;21(2):131-6.

19. Brzeski M, Madhok R, Capell HA. Evening primrose oil in patients with rheumatoid arthritis and side-effects of non-steroidal anti-inflammatory drugs. Br J Rheumatol. oct 1991;30(5):370-2.

20. Cleland LG, French JK, Betts WH, Murphy GA, Elliott MJ. Clinical and biochemical effects of dietary fish oil supplements in rheumatoid arthritis. J Rheumatol. oct 1988;15(10):1471-5.

21. Das Gupta AB, Hossain AKMM, Islam MH, Dey SR, Khan AL. Role of omega-3 fatty acid supplementation with indomethacin in suppression of disease activity in rheumatoid arthritis. Bangladesh Med Res Counc Bull. août 2009;35(2):63-8.

22. Dawczynski C, Schubert R, Hein G, Müller A, Eidner T, Vogelsang H, et al. Long-term moderate intervention with n-3 long-chain PUFA-supplemented dairy products: effects on pathophysiological biomarkers in patients with rheumatoid arthritis. British Journal of Nutrition. mai 2009;101(10):1517-26.

23. Dawczynski C, Hackermeier U, Viehweger M, Stange R, Springer M, Jahreis G. Incorporation of n-3 PUFA and Y-linolenic acid in blood lipids and red blood cell lipids together with their influence on disease activity in patients with chronic inflammatory arthritis - a randomized controlled human intervention trial. Lipids Health Dis. 4 août 2011;10:130.

24. Dawczynski C, Dittrich M, Neumann T, Goetze K, Welzel A, Oelzner P, et al. Docosahexaenoic acid in the treatment of rheumatoid arthritis: A double-blind, placebo-controlled, randomized cross-over study with microalgae vs. sunflower oil. Clinical Nutrition. 1 avr 2018;37(2):494-504.

25. Galarraga B, Ho M, Youssef HM, Hill A, McMahon H, Hall C, et al. Cod liver oil (n-3 fatty acids) as an non-steroidal anti-inflammatory drug sparing agent in rheumatoid arthritis. Rheumatology (Oxford). 1 mai 2008;47(5):665-9.

26. Geusens P, Wouters C, Nijs J, Jiang Y, Dequeker J. Long-term effect of omega-3 fatty acid supplementation in active rheumatoid arthritis. Arthritis \& Rheumatism. 1994;37(6):824-9.

27. Khaleghi E, Heidari P, Heidari B. Efficacy of fish oil in the treatment of patients with rheumatoid arthritis. Ann Rheum Dis $2005 ; 64$ (Suppl 3):448.

28. Kolahi S, Ghorbanihaghjo A, Alizadeh S, Rashtchizadeh N, Argani H, Khabazzi A-R, et al. Fish oil supplementation decreases serum soluble receptor activator of nuclear factor-kappa B ligand/osteoprotegerin ratio in female patients with rheumatoid arthritis. Clin Biochem. avr 2010;43(6):576-80.

29. Kremer JM, Lawrence DA, Jubiz W, Digiacomo R, Rynes R, Bartholomew LE, et al. Dietary fish oil and olive oil supplementation in patients with Rheumatoid Arthritis clinical and immunologic effects. Arthritis \& Rheumatism. 1990;33(6):810-20.

30. Kremer JM, Lawrence DA, Petrillo GF, Litts LL, Mullaly PM, Rynes RI, et al. Effects of high-dose fish oil on rheumatoid arthritis after stopping nonsteroidal antiinflammatory drugs clinical and immune correlates. Arthritis \& Rheumatism. 1995;38(8):1107-14.

31. Kremer JM, Bigauoette J, Michalek AV, Timchalk MA, Lininger L, Rynes RI, et al. Effects of manipulation of dietary fatty acids on clinical manifestations of rheumatoid arthritis. Lancet. 26 janv 1985;1(8422):184-7.

32. Kristensen S, Schmidt EB, Schlemmer A, Rasmussen C, Johansen MB, Christensen JH. Beneficial effect of n-3 polyunsaturated fatty acids on inflammation and analgesic use in psoriatic arthritis: a randomized, double blind, placebo-controlled trial. Scand J Rheumatol. janv 2018;47(1):27-36.

33. Leventhal LJ, Boyce EG, Zurier RB. Treatment of rheumatoid arthritis with gammalinolenic acid. Ann Intern Med. 1 nov 1993;119(9):867-73.

34. Madland TM, Björkkjaer T, Brunborg LA, Fröyland L, Berstad A, Brun JG. Subjective improvement in patients with psoriatic arthritis after short-term oral treatment with seal oil. A pilot study with double blind comparison to soy oil. J Rheumatol. févr 2006;33(2):307-10.

35. Magarò M, Zoli A, Altomonte L, Mirone L, De Sole P, Di Mario G, et al. Effect of fish oil on neutrophil chemiluminescence induced by different stimuli in patients with rheumatoid arthritis. Ann Rheum Dis. juill 1992;51(7):877-80.

36. Nielsen GL, Faarvang KL, Thomsen BS, Teglbjærg KL, Jensen LT, Hansen TM, et al. The effects of dietary supplementation with n-3 polyunsaturated fatty acids in patients with rheumatoid arthritis: a randomized, double blind trial. European Journal of Clinical Investigation. 1992;22(10):687-91. 
37. Nordström DC, Honkanen VE, Nasu Y, Antila E, Friman C, Konttinen YT. Alpha-linolenic acid in the treatment of rheumatoid arthritis. A double-blind, placebo-controlled and randomized study: flaxseed vs. safflower seed. Rheumatol Int. 1995;14(6):231-4.

38. Park Y, Lee A, Shim S-C, Lee JH, Choe J-Y, Ahn H, et al. Effect of n-3 polyunsaturated fatty acid supplementation in patients with rheumatoid arthritis: a 16week randomized, double-blind, placebo-controlled, parallel-design multicenter study in Korea. The Journal of Nutritional Biochemistry. 1 juill 2013;24(7):1367-72.

39. Sköldstam L, Börjesson O, Kjällman A, Seiving B, Åkesson B. Effect of Six Months of Fish Oil Supplementation in Stable Rheumatoid Arthritis. A Doubleblind, Controlled Study. Scandinavian Journal of Rheumatology. 1 janv 1992;21(4):178-85.

40. Sundrarjun T, Komindr S, Archararit N, Dahlan W, Puchaiwatananon O, Angthararak S, et al. Effects of n-3 Fatty Acids on Serum Interleukin-6, Tumour Necrosis Factor-a and Soluble Tumour Necrosis Factor Receptor p55 in Active Rheumatoid Arthritis. J Int Med Res. 1 oct 2004;32(5):443-54.

41. Sundström B, Stålnacke K, Hagfors L, Johansson G. Supplementation of omega-3 fatty acids in patients with ankylosing spondylitis. Scand J Rheumatol. oct 2006;35(5):359-62.

42. Tulleken JE, Limburg PC, Muskiet FAJ, Rijswijk MHV. Vitamin e status during dietary fish oil supplementation in rheumatoid arthritis. Arthritis \& Rheumatism. 1990;33(9):1416-9.

43. Veale DJ, Torley HI, Richards IM, O'Dowd A, Fitzsimons C, Belch JJ, et al. A double-blind placebo controlled trial of Efamol Marine on skin and joint symptoms of psoriatic arthritis. Br J Rheumatol. oct 1994;33(10):954-8.

44. Veselinovic M, Vasiljevic D, Vucic V, Arsic A, Petrovic S, Tomic-Lucic A, et al. Clinical Benefits of n-3 PUFA and 囚-Linolenic Acid in Patients with Rheumatoid Arthritis. Nutrients. 25 mars 2017;9(4).

45. Volker D, Fitzgerald P, Major G, Garg M. Efficacy of fish oil concentrate in the treatment of rheumatoid arthritis. J Rheumatol. oct 2000;27(10):2343-6.

46. Zurier RB, Rossetti RG, Jacobson EW, DeMarco DM, Liu NY, Temming JE, et al. gamma-Linolenic acid treatment of rheumatoid arthritis. A randomized, placebo-controlled trial. Arthritis Rheum. nov 1996;39(11):1808-17.

47. Taylor PC, Moore A, Vasilescu R, Alvir J, Tarallo M. A structured literature review of the burden of illness and unmet needs in patients with rheumatoid arthritis: a current perspective. Rheumatol Int. mai 2016;36(5):685-95.

48. Wen H, Ralph Schumacher H, Li X, Gu J, Ma L, Wei H, et al. Comparison of expectations of physicians and patients with rheumatoid arthritis for rheumatology clinic visits: a pilot, multicenter, international study. Int J Rheum Dis. août 2012;15(4):380-9.

49. Gossec L, Dougados M, Rincheval N, Balanescu A, Boumpas DT, Canadelo S, et al. Elaboration of the preliminary Rheumatoid Arthritis Impact of Disease (RAID) score: a EULAR initiative. Ann Rheum Dis. nov 2009;68(11):1680-5.

50. Clarke J, Herzberg G, Peeling J, Buist R, Corbett D. Dietary supplementation of omega-3 polyunsaturated fatty acids worsens forelimb motor function after intracerebral hemorrhage in rats. Exp Neurol. janv 2005;191(1):119-27.

51. Bhatt DL, Steg PG, Miller M, Brinton EA, Jacobson TA, Ketchum SB, et al. Cardiovascular Risk Reduction with Icosapent Ethyl for Hypertriglyceridemia. N Engl J Med. 3 janv 2019;380(1):11-22.

52. Rizos EC, Markozannes G, Tsapas A, Mantzoros CS, Ntzani EE. Omega-3 supplementation and cardiovascular disease: formulation-based systematic review and meta-analysis with trial sequential analysis. Heart. janv 2021;107(2):150-8.

53. Nicholls SJ, Lincoff AM, Garcia M, Bash D, Ballantyne CM, Barter PJ, et al. Effect of High-Dose Omega-3 Fatty Acids vs Corn Oil on Major Adverse Cardiovascular Events in Patients at High Cardiovascular Risk: The STRENGTH Randomized Clinical Trial. JAMA. 8 déc 2020;324(22):2268-80.

\section{Figures}




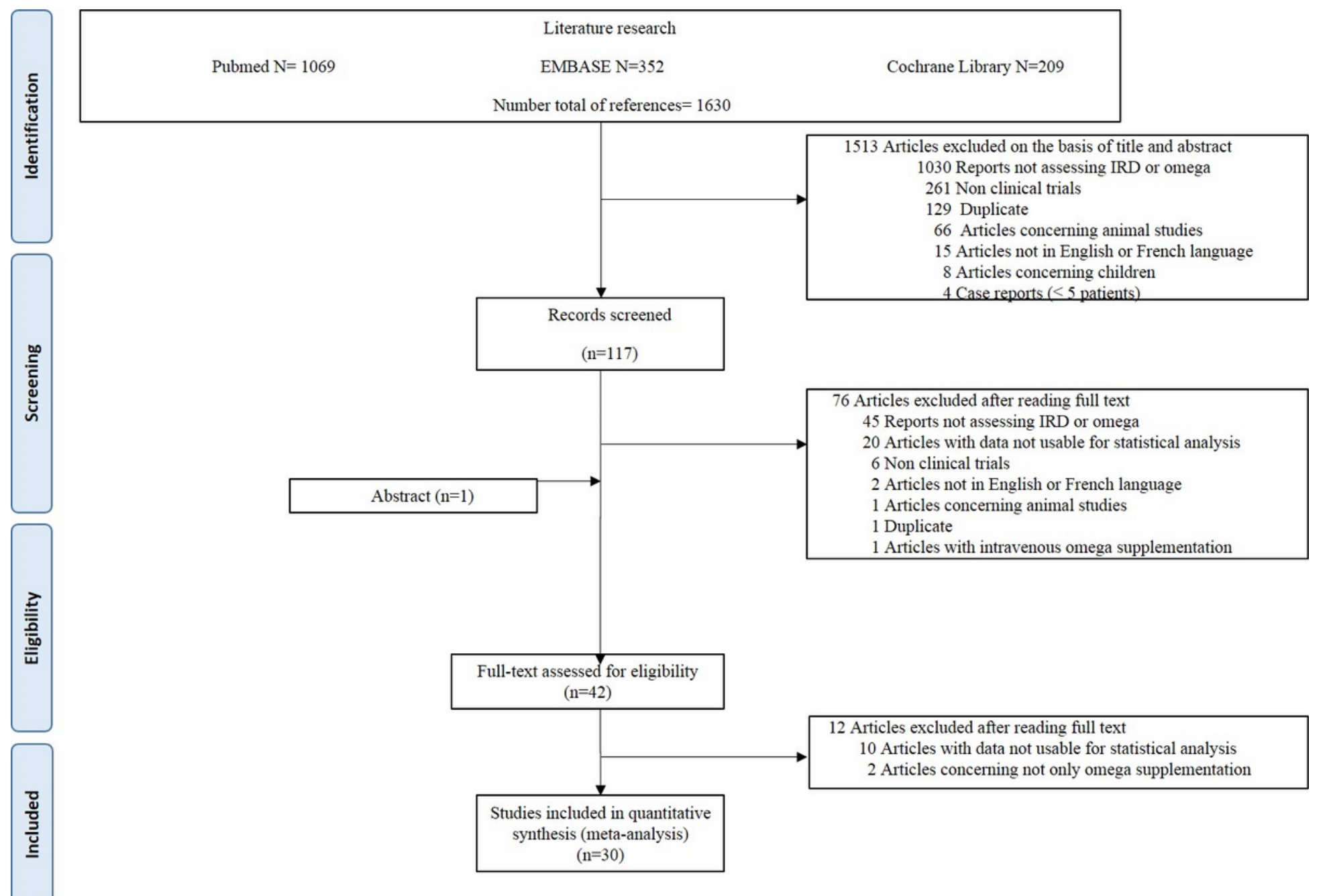

Figure 1

Flow diagram of the literature search process

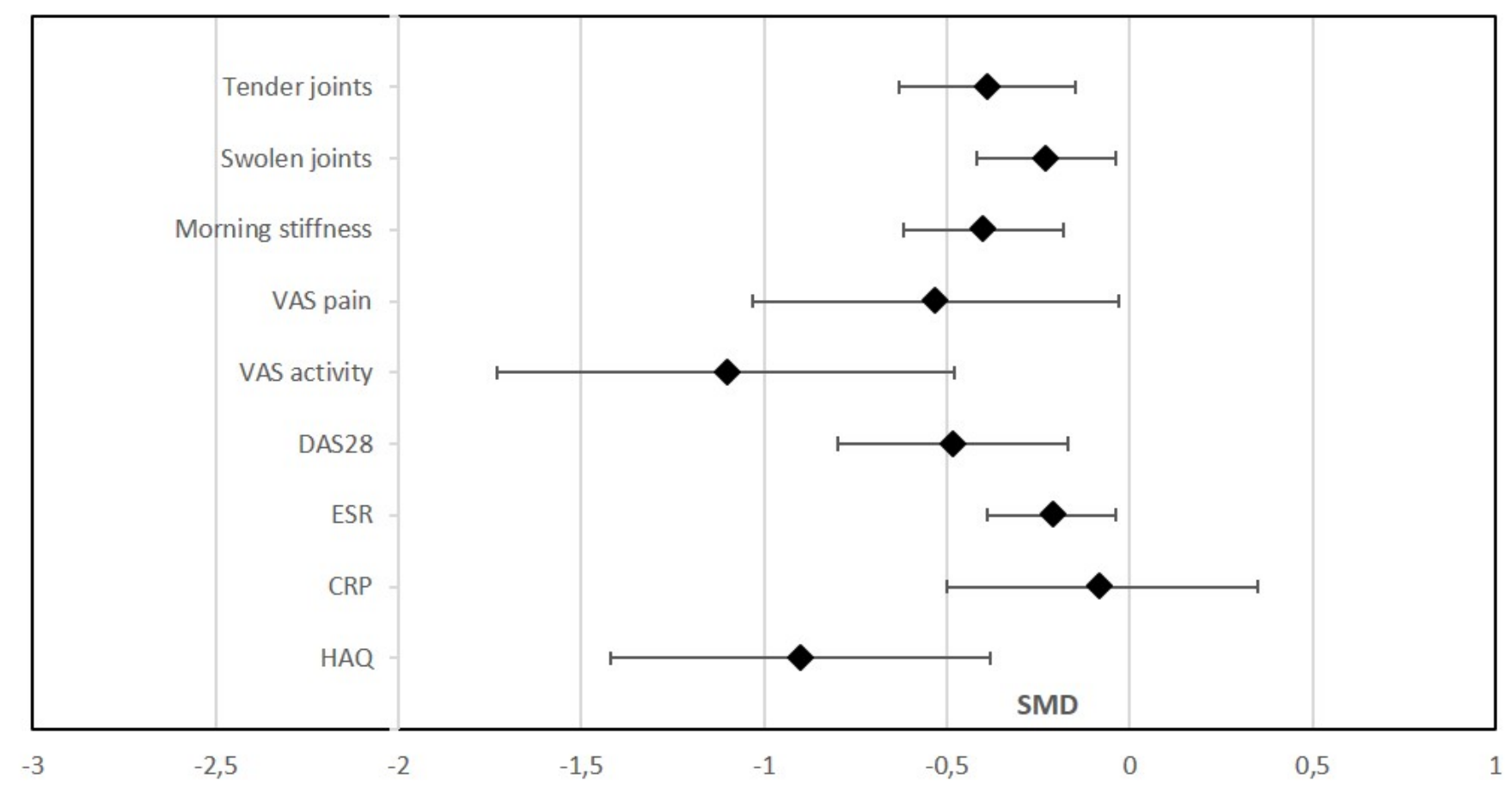

Page 12/14 
Figure 2

Overall effect of PUFA supplementation in rheumatoid arthritis (RA) patients compared to controls. VAS= visual analog scale; DAS28= Disease Activity Score in 28 joints; $\mathrm{ESR}=$ erythrocyte sedimentation rate; $\mathrm{CRP}=\mathrm{C}$-reactive protein; $\mathrm{HAQ}=$ Health Assessment Questionnaire
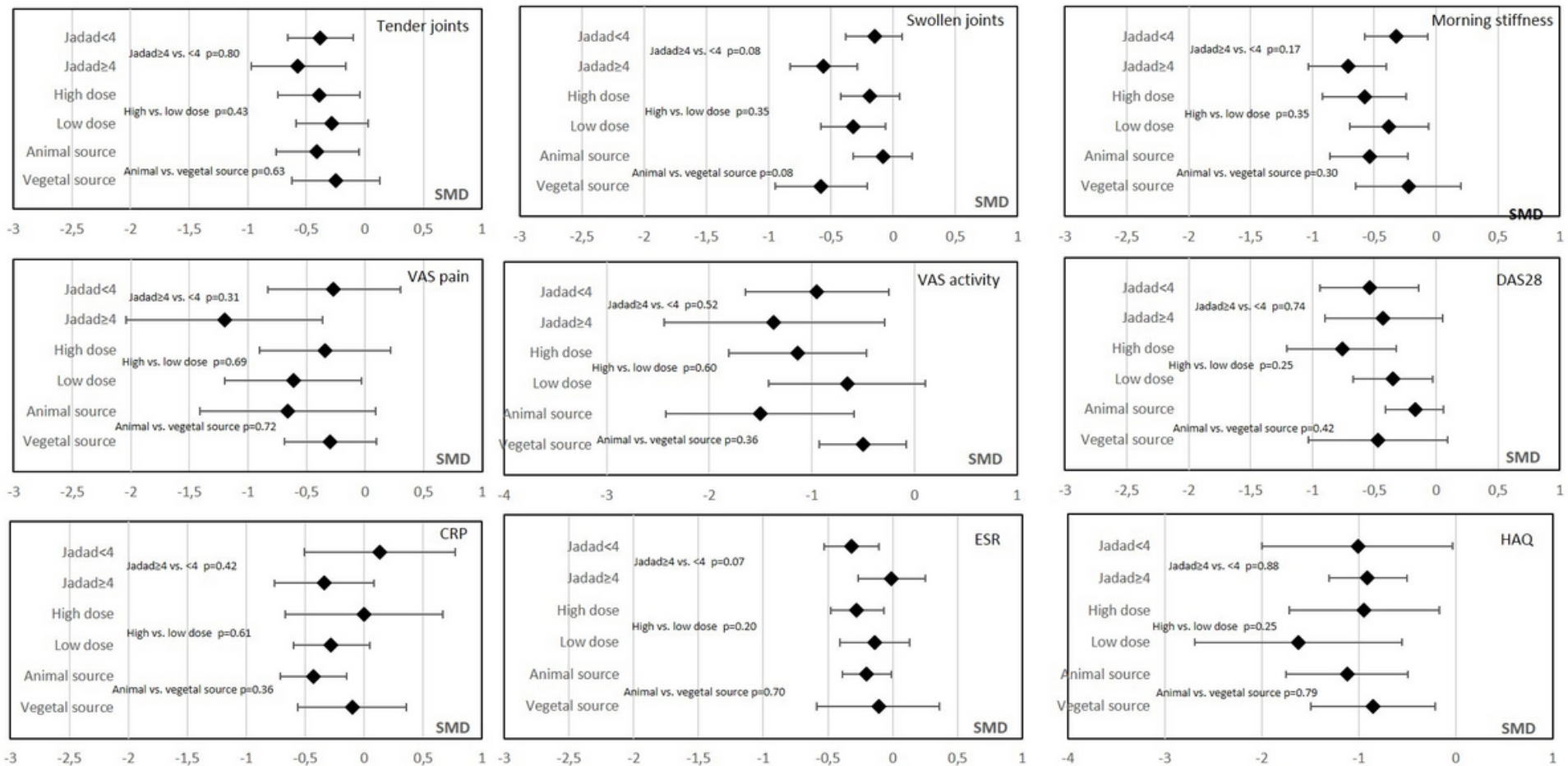

Figure 3

Meta-regression analysis of RA patients with oral PUFA supplementation. Data are standardized mean difference $(\mathrm{SMD})(95 \% \mathrm{Cl})$. VAS $=\mathrm{visual}$ analog scale; DAS28= Disease Activity Score in 28 joints; ESR= erythrocyte sedimentation rate; $C R P=$ C-reactive protein; HAQ= Health Assessment Questionnaire

$\mathrm{N}-3$ supplementation

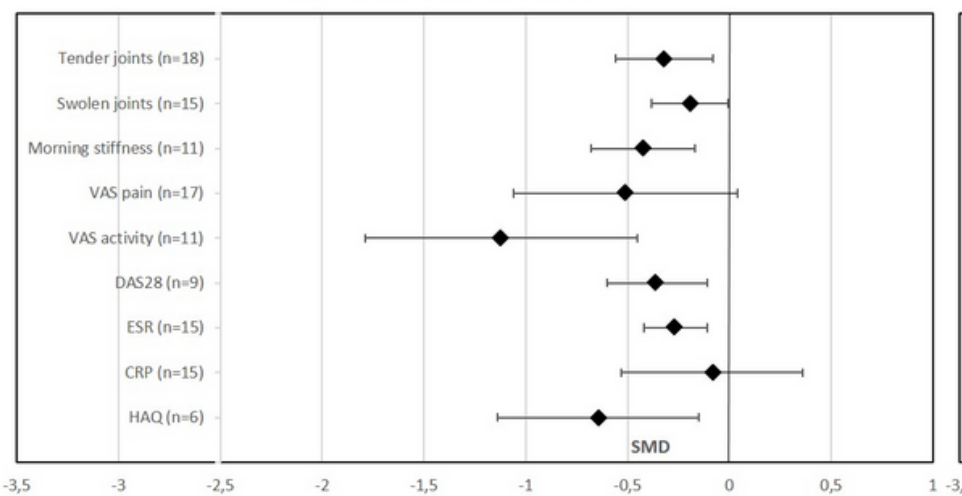

$\mathrm{N}-6$ supplementation

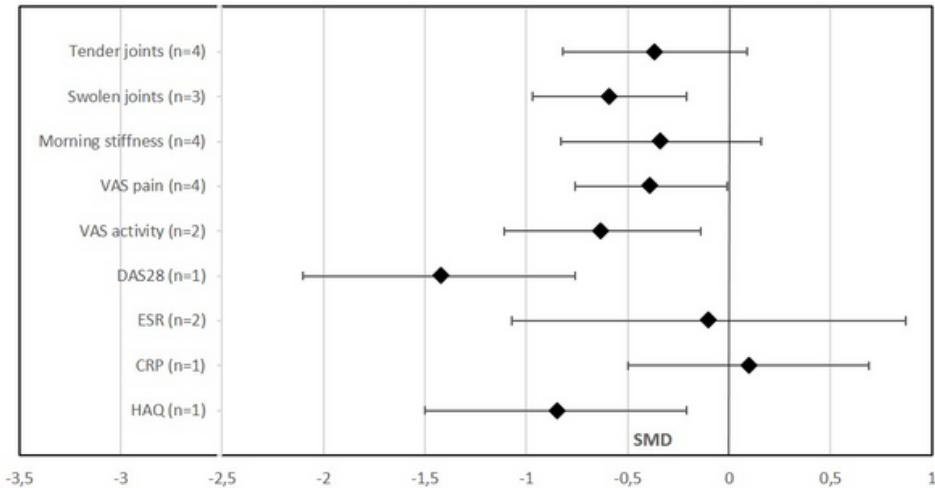

Figure 4

Effect of oral PUFA supplementation on RA disease activity by PUFA type ( $\mathrm{n}-3$ or $n-6)$. Data are standardized mean difference (SMD) (95\% Cl). VAS= visual analog scale; DAS28= Disease Activity Score in 28 joints; ESR= erythrocyte sedimentation rate; $C R P=C$-reactive protein; $H A Q=H e a l t h$ Assessment Questionnaire 


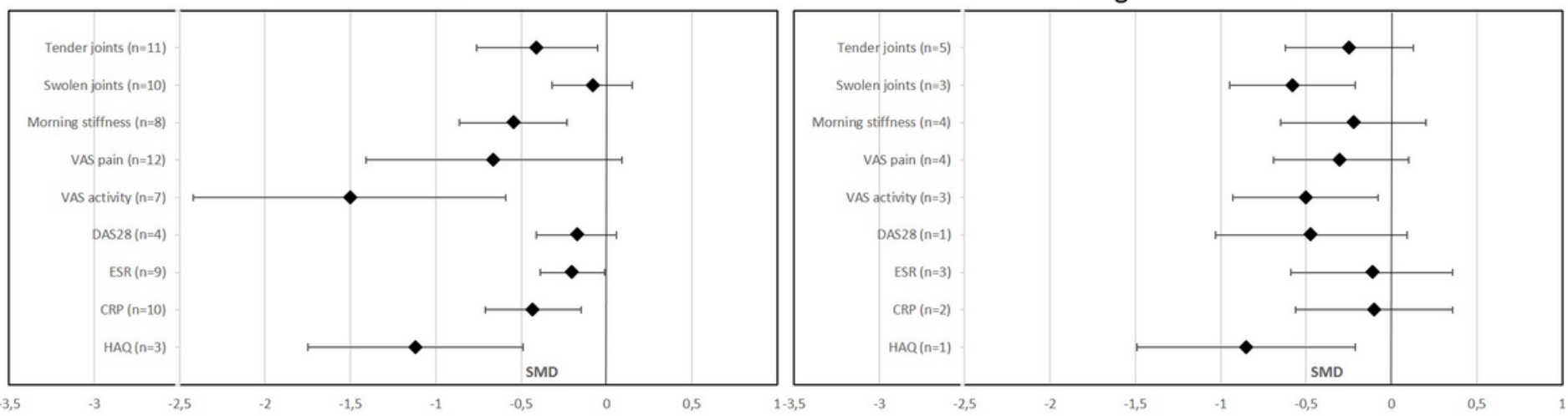

\section{Figure 5}

Effect of oral PUFA supplementation on RA disease activity by source (vegetable or animal). Data are standardized mean difference (SMD) (95\% CI). VAS= visual analog scale; DAS28= Disease Activity Score in 28 joints; $E S R=$ erythrocyte sedimentation rate; $C R P=C$-reactive protein; $H A Q=H e a l t h$ Assessment Questionnaire

Low dose

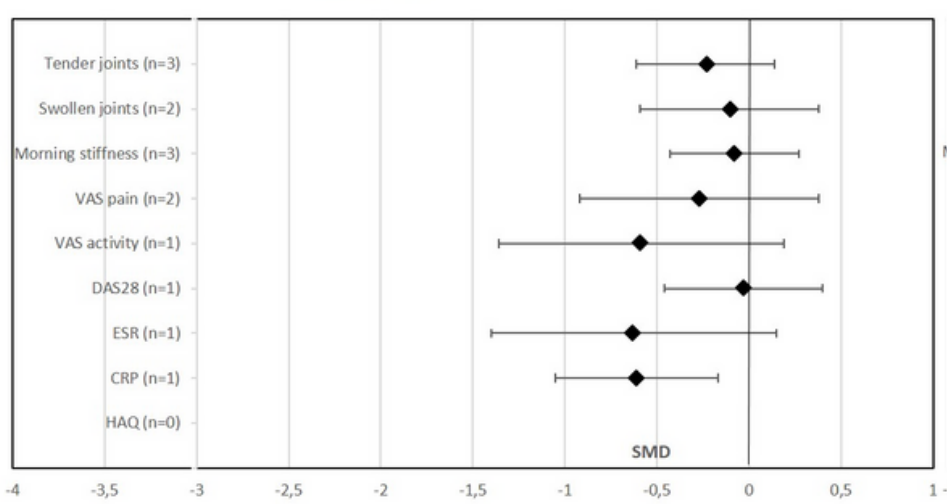

High dose

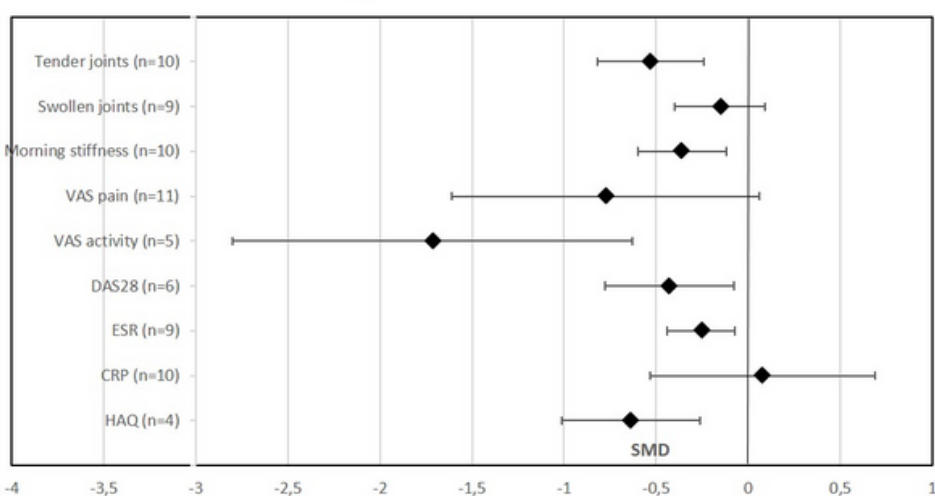

Figure 6

Effect of oral PUFA supplementation on RA disease activity by dosage ( $>$ or $<2 \mathrm{~g} /$ day $)$. Data are standardized mean difference $(\mathrm{SMD})(95 \% \mathrm{Cl})$. VAS $=\mathrm{visual}$ analog scale; DAS28= Disease Activity Score in 28 joints; $E S R=$ erythrocyte sedimentation rate; $C R P=C$-reactive protein; $H A Q=H e a l t h ~ A s s e s s m e n t$ Questionnaire

\section{Supplementary Files}

This is a list of supplementary files associated with this preprint. Click to download.

- Additionalfile1PRISMAChecklist.docx

- additionalfile2.docx

- additionalfile3.docx

- additionalfile4.docx

- additionalfile5.docx

- additionalfile6.docx

- additionalfile7.docx

- additionalfile8.docx

- additionalfile9.docx

- additionalfile10.docx

- additionalfile11.docx

- additionalfile12.docx

- additionalfile13.docx

- additionalfile14.docx

- additionalfile15.docx 